\title{
The future of measuring patient safety: prospective clinical surveillance
}

\section{Eric J Thomas}

Correspondence to

Dr Eric J Thomas, UT HoustonMemorial Hermann Center for Healthcare Quality and Safety, The University of Texas Medical School at Houston, 6410 Fannin UPB 1100.44, Houston, TX 77030, UK:

Eric.thomas@uth.tmc.edu

Received 16 February 2015 Accepted 16 February 2015

\section{SLinked}

http://dx.doi.org/10.1136/ bmjqs-2014-003432

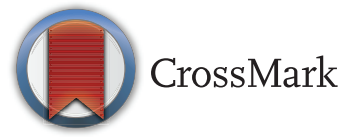

To cite: Thomas EJ. BMJ Qual Saf 2015;24:244-245.
Prospective clinical surveillance (PCS) is a safety measurement approach that fulfils many of the goals and principles of the latest thinking on measuring and improving safety. ${ }^{1} 2$ Compared with many current measurement practices, it may be more valid and reliable, with potential to facilitate learning and improvement and empower and draw upon the experience of front-line providers. It also has implications for external oversight of healthcare organisations.

In the study by Wong et $a l,^{3}$ PCS involved a trained nurse who visited a clinical unit on weekdays. The nurse looked for triggers indicating a possible adverse event by reviewing records of patients and talking to the front-line providers in the unit. The list of triggers used by the nurse was generated from prior research and input from providers in the unit, meaning that the triggers were customised for that unit. Trigger-positive records were then reviewed by an interprofessional team, which included frontline staff involved in the care, the trained observer and investigators. Providers were also encouraged to report additional events during this process.

In their 20-bed general medical ward in a tertiary care hospital, Wong et al found preventable harm in around $10 \%$ of patients. Adverse events included the usual suspects of adverse drug events, hospital acquired infections, falls, pressure ulcers and diagnostic errors. But other findings were perhaps more unexpected. Common categories of adverse events (eg, adverse drug events) comprised a very heterogeneous group of subtypes, and the number and type of contributing factors that led to the adverse events were quite heterogeneous too. The results imply that it will be difficult to improve overall safety by identifying and correcting very specific event types or by addressing just a few contributing factors common to many adverse events. The hard work of improving safety on this unit, and others, will instead involve many changes to many processes.

These findings present challenges for our current safety improvement approaches and infrastructure, but a difficult message is no reason to shoot the messenger. PCS has many real and potential advantages over the current methods of safety measurement which have wellknown flaws. ${ }^{4}$ Reporting systems do not provide a reliable estimate of event rates or types of events, ${ }^{4}$ and organisations can be over-or under-whelmed with reports from which no sense can be made. ${ }^{6} 7$ Retrospective chart review is timeintensive, relies on incomplete clinician documentation and gives little insight into latent organisational factors and contributing factors that lead to harm. Administrative data is for billing purposes, may not reflect actual clinical care and reveals very little about the causes of events. Triggers, as currently used, are typically applied long after the patient is gone, and they lead to time-consuming reviews of incomplete and inaccurate medical records. Direct observation of certain processes (eg, hand hygiene practices, dispensing or administering medications) is very time-intensive and may not provide information about why providers are committing errors or violating protocols. ${ }^{4}$

In contrast, PCS occurs in near real-time as the safety of care is being evaluated while the patient is still in the hospital. This has several advantages: they include making it easier for front-line providers to recall the details of the preventable adverse event, thus leading to a more accurate assessment of what went wrong and why. Prompt detection and correction of contributing factors could also prevent future events, while also enabling the amelioration of defects in near real time. PCS also has the ability to identify emerging harms that arise from the ever-changing and complex sociotechnical systems in which patients are cared for and that may be specific to that care setting. These new errors 
and harms can be detected and prevented by front-line providers engaged in identification and learning.

PCS relies on the knowledge of front-line providers by drawing upon their experience to create the triggers. Furthermore, they participate on the interdisciplinary committee that judges preventability of the adverse events and identifies contributing factors. This degree of participation is unusual in most healthcare organisations, but is a fundamental tenet of safety and quality improvement in other fields. In addition to improving the validity of the data collected, front-line provider involvement can improve safety culture by engaging more staff in reporting, analysing and learning from preventable adverse events. Doing this has the potential to improve employee engagement and job satisfaction, which has also been tied to better organisational performance. ${ }^{8}$

PCS highlights the limitations of publically reported safety data. Mandated, externally reported adverse events represent a small fraction of the all the harm that occurs in hospitals. However, hospitals are incentivised to focus on these events, resulting in tunnel vision, and a false sense of security if rates of those selected events improve. ${ }^{9}$ PCS could open the eyes of hospital leaders and their external overseers to the true frequency, heterogeneity and severity of harm occurring to their patients. PCS might help clarify the breadth and depth of safety issues in organisations, limiting the temptation to present a limited set of publically reported measures and declare success.

PCS also fulfils many of the proposed guiding principles of safety measurement. ${ }^{1}{ }^{2}{ }^{4}$ It recognises that there is no single measure (nor single measurement method) of or for safety. It requires more provider participation and more organisational commitment than most existing measures, thus increasing the visibility of safety within an organisation. It is unit-based, thus recognising that there is no single overall measure of safety for a hospital. And it empowers the front-line caregivers and encourages learning on a day-to-day basis.

For PCS to meet its potential, several things will be needed. Front-line providers will need more training in safety and implementation science so they can understand systems, identify hazards, develop triggers and contribute to improvement efforts. The reliability of the method will need to be improved before it can be used to assess if safety is improving or not. Leaders will need to improve their safety culture so that the providers feel comfortable speaking up. Experts in relevant safety sciences will need to be available to advise the unit-based interdisciplinary groups that are judging preventability and identifying contributing factors. Information technology will need to be used to facilitate event detection. ${ }^{10}$ And finally, hospitals will need to have organizational structures in place to share lessons learned among different clinical areas.

The limitations of using a few safety measurement methods isolated from one another and overseen by so-called experts who are detached from daily patient care have been known for many years. Now that these researchers and front-line providers have demonstrated how PCS can be used, and given its potential advantages over the status quo, it is time to stop relying solely on traditional patient safety measurement approaches and to expand, evaluate and improve PCS to measure patient safety.

Competing interests None.

Provenance and peer review Not commissioned; internally peer reviewed.

\section{REFERENCES}

1 Berwick DM. A promise to learn-a commitment to act. Improving the safety of patients in England. London: Department of Health, 2013.

2 Vincent C, Burnett S, Carthey J. Safety measurement and monitoring in healthcare: a framework to guide clinical teams and healthcare organisations in maintaining safety. BMJ Qual Saf 2014;23:670-7.

3 Wong BM, Dyal S, Etchells EE, et al. Limitations associated with intensive adverse event measurement to inform quality improvement strategies: implications for trigger tool methodology. BMJ Qual Saf 2015;24:272-81.

4 Thomas EJ, Petersen LA. Measuring errors and adverse events in healthcare. J Gen Intern Med 2003;18:61-7.

5 Pronovost PJ, Miller MR, Wachter RM. Tracking progress in patient safety. An elusive target. JAMA 2006;296:696-9.

6 Shojania KG. The frustrating case of incident-reporting systems. Qual Saf Health Care 2008;17:400-2.

7 Wachter RM. Understanding patient safety. 2nd edn. McGraw Hill, 2012.

8 Etchegaray JM, St. John C, Thomas EJ. Measures and measurement of high-performance work systems in healthcare settings: propositions for improvement. Health Care Manage Rev 2011;36:38-46.

9 Thomas EJ, Classen DC. Patient safety: let's measure what matters. Ann Intern Med 2014;160:642-3.

10 Bates DW, Evans RS, Murff $\mathrm{H}$ et al. Detecting adverse events using information technology. J Am Med Inform Assoc 2003;10:115-28. 